\title{
Coordination in gait: Demonstration of a spectral approach
}

\begin{abstract}
Many important notions in Life Sciences are linked with the idea of cycles, periodicity, fluctuations and transitions. The aim of this paper is to use spectral analysis in a unique way to study and quantify whole body coordination during gait. A participant walked at $3 \mathrm{~km} / \mathrm{h}$ and ran at $15 \mathrm{~km} / \mathrm{h}$ on a treadmill for 2 minutes. Position of the approximate center of rotation of the toe, ankle, knee, hip, shoulder, elbow and wrist, and heel, PSIS and head were collected at $100 \mathrm{~Hz}$ using CODAmotion analysis system. Fast Fourier Transform was performed on $\mathrm{x}$-coordinate data of the 1) knee marker; 2) 4 markers attached to the free lower limb (toe, ankle, heel and knee); 3) left and right free lower limbs; 4) whole body (all markers). Gait is described by a largely harmonic and resonant oscillator that operates unilateral free limbs at the stride frequency, and axial regions at the step frequency. Running is described by a more harmonic and resonant oscillating structure than walking, with a 3 times higher Q factor and $47 \%$ lower Inharmonicity Index. This method is presented as a way to capture global dynamics of our complex multi-segment system, and presents a novel application of spectral analysis to study coordination in multiple oscillators.
\end{abstract}

Keywords: gait analysis, dynamical systems theory, phase relations, coherence, biomechanics.

\section{Introduction}

Many important notions in Life Sciences are linked with the idea of cycles, periodicity, fluctuations and transitions; from the thermodynamic and chemical processes underpinning the function of a cell, to the modes of gait used for locomotion. Furthermore, in biological systems, the interaction of many linked oscillatory parts, and the redundancy afforded by their coordination underpins the functionality and health of that system. For example, understanding coordination between multiple moving segments of the body, and particularly the regularities and variations in this coordination, is key to understanding motor control from a dynamical systems theory perspective (Bernstein, 1967; Haken, Kelso, \& Bunz, 1985; Kamm, Thelen, \& Jensen, 
1990; Kelso, 1995; Kugler, Kelso, \& Turvey, 1980; Newell, 1989). For example, coordinative structure theory and the Haken, Kelso and Bunz (HKB) Model (Haken, Kelso, \& Bunz, 1985; Kelso, 1995; Kugler, Kelso, \& Turvey, 1980) originally examined the couplings and phase relations between two individual oscillating fingers. Based on observations of multi-stability in phase relations, non-linear phase transitions and hysteresis, the work of Haken, Kelso and Bunz (1985) provided theoretical and empirical evidence that coordination in a biological system is an emergent selforganised process. However, there are still few examples of coordinative structure theory being applied to whole body actions such as gait.

Bernstein's (1967) problem seeks to understand how the many degrees of freedom are organized to master the system redundancy. However, measures of coordination used in motor control and biomechanics predominantly consider phase relations between only two oscillators, through measures of Relative Phase, Vector Coding and Continuous Relative Phase (Costa, Golderberger, \& Peng, 2005; van Emmerik et al., 2014; van Emmerik et al., 2016; von Holst, 1954; Scholz, Kelso, \& Schöner, 1987). Alternative approaches describe the state of the entire system through phase relations in two biomechanically relevant global variables such as the centre of mass and centre of pressure (Newell et al., 2015; Segers et al., 2007; Vereijken et al., 1992). Statistical methods such as Principal Component Analysis have been used to capture the relations between multiple degrees of freedom to reduce the dimensionality of data (Daffertshofer et al., 2004; Lamoth et al., 2009), increasing our understanding of the coordination involved in whole body tasks. However, human movement research is still searching for ways to capture global dynamics of our complex multi-segment system. Gait involves multiple moving segments and is cyclic in nature. Gait modes for humans fall into two categories; walking and running, for which biomechanical (Alexander, 
2004; Biewener et al., 2004; Farley \& Ferris, 1998; Lee \& Farley, 1998; Novacheck, 1998; Segers et al., 2006, 2007a, b) and motor control (Bohnsack-McLagan, Cusumano, \& Dingwell, 2016; Daffertshofer et al., 2004; Dingwell \& Cusumano, 2015; Lamoth et al., 2009; Li et al., 1999; Li, Haddad, \& Hamill, 2005; Seay et al., 2006; Schöner, Jiang, \& Kelso, 1998) aspects have been studied over decades. Previous studies have analysed symmetry within a stride, and stride length in gait (Gage, 1964; Roche at al., 2013; Smidt et al, 1971; Wagenaar, \& Van Emmerik, 1994; 2000), however previous work has not considered how the combination of oscillations of different segments create a global dynamic.

Since Fourier transform is a way to study the frequency content of a signal, looking at the structure of the regular phenomena involved, it allows us to unravel the complexity and dimensionality of the combined oscillations. By understanding how the components within the system are interacting with each other we can further understand how the system works on a global level and provide a holistic view of the whole body movements. To provide a basic demonstration of how spectral analysis can capture coordination, we firstly analysed data of a bimanual coordination task (Haken, Kelso, \& Bunz, 1985). Kinematic data (CODA, Charnwood Dynamics Ltd, UK; 100 Hz) were collected from 2 markers placed on the distal phalange of each index finger as a participant, beginning in an anti-phase mode, increased the velocity of abductionadduction finger oscillation with a constant acceleration. Figure $1 \mathrm{a}$ and $1 \mathrm{~b}$ show the spectrogram of the rhythmical oscillation of the index finger on each hand. For each finger (Figure 1a and 1b) the base frequency (vertical axis) increased over time (horizontal axis) as the speed of the finger oscillation increased. Figure 1c shows the spectral result of adding the signals of finger 1 and finger 2. At velocities where antiphase oscillations between fingers occur, the spectrum shows the effect of the 
destructive interference between the two waveforms, cancelling the base frequency between $0-45 \mathrm{~s}$. As the fingers transition into in-phase coordination there is first a blur in the spectrum that represents the out-of-phase and phase variability associated with phase transitions, then, as the fingers lock into an in-phase coordination from 50-60 s, the spectral line associated with their oscillation is enhanced, as a result of constructive interference.

Using spectrograms we can obtain an individual and situation specific representation of coordination by examining movement of multiple markers that represent moving segments of the body. Measuring, for example, the displacement of each joint centre while walking or running, provides a series of one-dimensional oscillating waveforms. The collection of these waveforms, their frequencies, amplitudes, and phase-relations captures the coordinated movement of the whole body. The aim of this paper is to demonstrate the use of spectral analysis as a unique way to study coordination during gait through the analysis of constructive and destructive interference.

\section{Methods}

\section{Data collection}

Ethical approval was gained from the Department of Life Sciences Ethics Panel at Anglia Ruskin University. Informed consent was gained from the participant prior to testing. A male participant (age 28 years, mass $75.2 \mathrm{~kg}$, height $1.90 \mathrm{~m}$, regular runner $\sim 45$ mins $\mathrm{x} 4$ times per week) walked and ran on a motorized treadmill at constant speeds of 3 and $15 \mathrm{~km} / \mathrm{h}$ for 2 minutes. Five minutes rest was provided between each 
speed to mitigate the effects of fatigue. Kinematic data were collected during all gait trials using an automated 3D motion capture system (CODA, Charnwood Dynamics Ltd, UK) sampling at $100 \mathrm{~Hz}$. Three CX1 CODA scanners provided a field of view exceeding $2.5 \mathrm{~m}$ around the treadmill. CODA was aligned such that the $\mathrm{x}$ axis was the direction of travel of the participant. 22 active markers were placed bilaterally on the lateral aspect of the estimated centre of rotation of the shoulder, lateral epicondyle of the humerus, head of the ulna, estimated centre of rotation of the fifth metacarpophalangeal joint, temple, posterior superior iliac spine (PSIS), greater trochanter, femoral condyle (knee), lateral malleolus (ankle), fifth metatarsophalageal joint, calcaneus (heel) and the first distal phalanx of the foot (toe).

\section{Data processing}

Positional data of markers and combinations of markers in the direction of travel ( $\mathrm{x}$ direction) were analysed using R (http://www.r-project.org) with the seewave package (http://rug.mnhn.fr/seewave/) to perform Fast Fourier Transform (FFT) with Hanning window. Spectrograms were computed using 216 point moving Hanning windows with no overlap for each marker and combination of markers. Through an additive approach, Fourier theorem provides a way to weight the contribution of each frequency component through their relative amplitude. Four combinations of $\mathrm{x}$ direction (sagittal plane) data were analysed using the FFT: 1) knee marker; 2) four markers attached to the left free lower limb (knee, ankle, heel and toe); 3) all eight markers of the left and right free lower limbs; 4) whole body (all 22 markers). When required, for example to allow comparison between running speeds, the spectra were normalized to the base frequency.

Discrete variables; base frequency, Q Factor and Inharmonicity Index were calculated from the Fourier spectra. Base frequency was calculated from the position of the 
fundamental frequency peak in the spectrum. The Q Factor is the resonance quality factor of a frequency spectrum and was determined using the $\mathrm{Q}$ function in seewave which estimates the frequency pureness of a time wave by returning the resonant quality factor at a specific $\mathrm{dB}$ level $(-3 \mathrm{~dB})$. The Inharmonicity Index is a quantitative description of how a certain spectrum was deviating from a perfectly harmonic one; calculated based as the average of the exponential of the deviation of a peak from a perfectly harmonic spectrum (Equation 1). By definition it is always greater or equal to 0 , where 0 is a purely harmonic spectrum as the overtones are integer multiples of a fundamental frequency; the larger the value the more inharmonic the spectrum. Equation 1:

$$
I=\frac{\sum_{i} e^{\left|f / f_{1}-i\right|}}{N}-1
$$

Where: $I$ is the Inharmonicity Index, $N$ is the number of spectral components, $f_{i}$ is the frequency of the $i$-th peak in the spectrum, $f_{l}$ is the fundamental frequency. For example, for an almost perfectly harmonic spectrum with four spectral peaks at: 100, 202, 299, 402, the Inharmonicity Index would be:

$$
I=\frac{e^{|100 / 100-1|}+e^{|202 / 100-2|}+e^{|299 / 100-3|}+e^{|402 / 100-4|}}{4}-1=0.0076
$$

On the other hand, if the peak frequencies are further apart from the harmonic sequence, for example: 100, 215, 312, 422, the Inharmonicity Index would be more than 17 times higher:

$$
I=\frac{e^{|100 / 100-1|}+e^{|215 / 100-2|}+e^{|312 / 100-3|}+e^{|422 / 100-4|}}{4}-1=0.134
$$




\section{Results}

Figures 2 and 3 represent the spectrograms of the four different marker combinations for both walking at $3 \mathrm{~km} / \mathrm{h}$ and running at $15 \mathrm{~km} / \mathrm{h}$. The spectrogram offers an easily interpretable map of how the spectrum evolves, thus revealing the stability of the frequency relations. Amplitude related to each frequency component (vertical axis) is represented by colours which change over time (horizontal axis). Figure 2 and 3 qualitatively express phase cancellations and enhancements as a result of combining markers.

INSERT FIGURE 2 HERE

\section{Walking}

Figure $2 \mathrm{a}$ is the spectrogram of the waveform of the knee marker in the $\mathrm{x}$ direction (sagittal plane) as the participant walks at $3 \mathrm{~km} / \mathrm{h}$. Due to the cyclical nature of the oscillation, the spectrum is clearly structured, fundamentally harmonic with clear spectral lines appearing at frequencies corresponding to integer multiples of the base frequency. The base frequency, where most of the energy is concentrated, is $0.78 \mathrm{~Hz}$, which is $9.3 \%$ higher than the stride frequency $(0.71 \mathrm{~Hz} \pm 0.02)$ seen as a red (high amplitude) spectral line in Figure 2a. There is an overall background noise due to unstructured characteristics of the signal. The consistency of the spectral lines in the horizontal axis demonstrates that this is a stable action occurring over time.

By adding the toe, ankle and heel markers to the knee marker (Figure 2b) the Inharmonicity index decreases (Table 1), showing a fundamentally harmonic spectrum with most of the energy concentrated around the stride frequency. Compared to Figure 
$2 \mathrm{a}$, the amplitude of the harmonics are reduced due to the phase oppositions that exist between movements of the leg markers.

Figure $2 \mathrm{c}$ is the spectrum of the waveform created by combing the left and right legs (Leg-Leg). The spectrum is made of two key contributions, the first at the step frequency concentrated at $1.37 \mathrm{~Hz}$, which is 1.93 times the stride frequency and the second at the first harmonic of the step frequency at $2.73 \mathrm{~Hz}$. Interestingly, the fundamental frequency for the leg-leg spectrum has become the step frequency, rather than the lower stride frequency for the knee (Figure 2a) and the individual leg (Figure 2b). The stride frequency has been cancelled due to the anti-phase movements of the legs. Inharmonicity Index of this combination is 0.102 , representing a further tendency towards inharmonic as a result of the additional phase delays and path differences between opposing limbs in walking. Q factor of the resonance increases compared to single leg analysis (Table 1).

Figure $2 \mathrm{~d}$ is the spectral result of the combination of all 24 markers. The spectrum is fundamentally harmonic, with the main contribution coming from $1.367 \mathrm{~Hz}$, which is 1.93 times the stride frequency (Table 1). The value of the Inharmonicity Index is 0.063 and the $\mathrm{Q}$ factor is 3.83, due to the more coherent phase relations and constructive interference resulting from the combination of whole body markers. Pairing of inertial components in the contralateral limbs has led to the strongest cancellation of all other spectral lines. 


\section{Running}

Figures 3a-3d are the spectrograms of the same marker combinations (knee, leg, LegLeg and whole body) for the participant running at $15 \mathrm{~km} / \mathrm{h}$. The knee and the leg spectra (Figure 3a, b) show a fundamentally harmonic spectrum with most of the energy concentrated around $1.37 \mathrm{~Hz}$ (5.5\% less than the stride frequency). The main difference between the two is the second harmonic disappearing and the fourth appearing in Figure $3 \mathrm{~b}$, as a result of specific phase relation between the segments of the leg. The Inharmonicity Index is 0.007 , close to a perfectly harmonic one. Q factors are 5.11 for the knee and 5.12 for the leg, higher than those related to the knee oscillation during walking.

Figure $3 \mathrm{c}$ is the spectrum of the waveform created by combing the left and right legs while running. Stride frequency characteristics are cancelled between the two legs due to anti-phase movements, leaving the step frequency as dominant. A Q factor of 10.31 suggests a high resonance for this combination, 3.39 times higher than the one in walking, where the spectrum is also more harmonic.

Finally, Figure $3 \mathrm{~d}$ is the spectrogram of the combination of all body marker oscillations in the $\mathrm{x}$ direction as the participant runs at $15 \mathrm{~km} / \mathrm{h}$. The spectrum is the most harmonic, $47 \%$ lower than that of walking. Most of the energy at the base frequency of $2.93 \mathrm{~Hz}$, which is the step frequency. Q factor is 2.93 times higher than the whole body $\mathrm{Q}$ factor in walking showing an overall increase in stability compared to walking. Interestingly, compared to the leg-leg combination, the sub-harmonic (the stride frequency) has been strengthened by the addition of the whole body markers, which is due to the head and 
trunk (as represented by the temple, estimated centre of rotation of the shoulder, PSIS and greater trochanter) oscillating at the stride frequency.

\section{INSERT TABLE 1 HERE}

INSERT FIGURE 4 HERE

Figure 4 is the spectrum of the whole body marker combination having been normalized to the base frequency for both walking $(3 \mathrm{~km} / \mathrm{h})$ and running $(15 \mathrm{~km} / \mathrm{h})$. This figure emphasizes the amplitude of the frequencies having been enhanced or cancelled, and the harmonic structure of the spectra presented as how the peaks lay according to integer multiples of 1 . Walking at $3 \mathrm{~km} / \mathrm{h}$ has a drift from a perfectly harmonic behaviour (0.063), whereas running at $15 \mathrm{~km} / \mathrm{h}$ is strongly harmonic $(0.001)$.

INSERT FIGURE 5 HERE

Figure 5 shows the frequency distribution of the spectrum in $2 \mathrm{~Hz}$ bands. There is a maximum difference of $0.4 \%$ between the walking and running spectrum in band 0-2 $\mathrm{Hz}$, demonstrating that this method does not clearly distinguish between the spectrum for walking and running.

\section{Discussion}

This paper presents spectrograms and spectral analysis as a way to study whole body coordination in walking and running gaits. Specifically, the spectral result of combined marker oscillations that describe whole body movements was used to further understand 
the collective behaviour, or global dynamic, of our biomechanical system. The final result of the interaction of all markers is a globally simpler dynamical system, described by a smaller number of frequencies (Figure 2 and 3). Therefore, this approach initially moves in the opposite direction to that of standard spectral analysis; allowing the global variable to emerge through the determining the frequency of the emergent global variable rather than decomposing signals into its frequency content, then decomposes the frequency content of the resulting signal. The frequencies themselves, their spread and relations provide information about the coordinated nature of the system during walking and running gaits.

Previous studies have analysed harmonic theory in human movement science, for example to examine the symmetry within a stride by exploiting the periodicity of the signal (Gage, 1964; Roche at al., 2013; Smidt et al, 1971). Building on this work, the current technique examines phase relations between the oscillating degrees of freedom of the body segments. Adding to the analysis of asymmetry and coordination analysis using PCA (Daffertshofer et al., 2004; Lamoth et al., 2009), the current technique embraces the expression of the mechanical phase relations that have evolved to underpin gait through relative inertias, delays, frequency enhancement and cancellation; i.e. the body's mechanical solution over steps and time. For example, in running, comparing the whole body marker combination (Figure 3d) and the left and right leg combination (Figure 3c), the first harmonic has been cancelled due to leg-arm coordination. The contribution at the base frequency has reduced due to the destructive interference of anti-phase components capturing the whole body mechanical solution to running. The tightness of the anti-phase coupling is related to the purity of the cancellation of the base frequency, which is represented by the Q factor. 
Furthermore, from a quantitative perspective, the measures of Q factor and Inharmonicity Index provide a description of the profile of the spectrum rather than a statistical breakdown of its content. For example, Figure 5 shows the frequency distribution of the spectrum in $2 \mathrm{~Hz}$ bands, a technique which is often used in motor control research to distinguish data. It is clear that with a maximum difference of $0.4 \%$, this representation of the spectrum does not provide enough information about the quality of the coordination that is conveyed through the spectrum in Figure 4. Furthermore, through quantitative analysis of the spectrum, the quality of the coordination is related to the shape of the peaks in the spectrum (the height and width: Q factor) and the position (Inharmonicity Index). Therefore, summarizing the spectrum or the spectrogram using bands masks the important information that characterizes coordination.

Through the analysis of spectrograms it is clear that gait is described by a largely harmonic and resonant oscillator that operates unilateral free limbs at the stride frequency, and axial regions at the step frequency. Running is described by a more harmonic combination of oscillations which has a stronger resonance than walking. In fact, this is true for the whole system, as well as limbs and cross limb pairs. The Q factor measures frequency dispersion around a central frequency, in this case a peak in the amplitude, and is a measure of the resonance quality in the combination of oscillations. Q factor was 2.94 times higher in running compared to walking, showing a lower dispersion of frequencies around the central peak values. Therefore, the overall coordination of the system during running is more phase coherent, efficient, stable, and less susceptible to perturbations when running. While these inferences are based on the mechanics of oscillators, they are of relevance to motor control where stability of the global dynamic has been defined based on phase coherence, reduced variability (phase 
dispersion) that result in a more stable attractor (van Emmerik et al., 2016; Haken et al., 1985). This finding is in line with those of Lamoth et al. (2009), who used a PCA to demonstrate that phase relations changed and coupling strengthened from walking to running; with the most prominent movements becoming more tightly coupled.

From a biomechanical perspective efficiency (Cavagna and Kaneko, 1977) and stability are key if the gait speed is near the maximum limit due to the high level of constraints placed on the degrees of freedom. The Q factor and the Inharmonicity Index are directly related to the efficiency of the system (Feynman et al., 1989); the Q factor is also related to its stability. This is demonstrated here as running has a higher Q and a lower Inharmonicity Index, suggesting it is a more efficient mechanical action, with more energy required to perturb it. On the other hand, while running is less prone to perturbations than walking, if any change to the pattern of coordination is required, to change direction for example, the mechanical system needs to expend more energy compared to walking to enable this.

Inharmonicity Index, based on its calculation, is independent of Q factor since it takes into account the position of the peaks not their width and amplitude. Inharmonicity is a measure of the deviation from a perfectly harmonic spectrum, which contains only integer multiples of a fundamental frequency. All natural oscillating phenomenon deviate from perfectly harmonic (Feynman at al., 1989a). Inharmonic frequencies are caused by combining anthropometrically different oscillating structures. From a motor control perspective, Inharmonicity can be considered a measure of complexity; the closer to 0 the Inharmonicity, the more harmonic, hence simpler is the global dynamic. Deviating from 0 means that independent oscillators contribute to the coordination, increasing the number of active dynamic degrees of freedom needed to recreate the movement pattern. Based on this proposition, increasing constraints from walking to 
running induces the simpler collective description of the coordination from the spectral point of view (Figure 2 and 3; Table 1), where the Inharmonicity Index of the whole body is $47 \%$ lower in running compared to walking. Therefore it suggests that the pattern of movement in running is easier to control and maintain since it occurs after the critical point in the control parameter; gait velocity (Schöner \& Kelso, 1988). From a basic and applied science perspective, future work will explore how Inharmonicity changes with pathology, age and injury in line with the loss of complexity hypothesis and interruptions to neural networks and pattern generators (Guertin, 2012; Lipsitz, 2002; Lipsitz and Goldberger, 1992; Schöner, Jiang and Kelso, 1990; Schöner and Kelso, 1988).

The global result of oscillations is more harmonic and phase coherent than that of the individual elements, suggesting there is evidence supporting an overall pattern generator based on one single oscillator (Q and Inharmonicity; Guertin, 2012; Schöner, Jiang, \& Kelso, 1990; Schöner \& Kelso, 1988). From a dynamical system perspective, the global dynamic created by the combination of all of the oscillators could be a candidate collective variable for gait (Newell \& Vaillancourt, 2001; Zanone \& Kelso, 1997). Therefore, this will open the field to further theoretical investigation of this phenomenon in line with the tenants of self-organization. In addition, spectrograms can provide practitioners with non-numerical tools for investigating coordination and stability over time.

\section{Conclusions}

This paper presents spectrograms and spectral methods to study whole body coordination in walking and running gaits through the analysis of constructive and destructive interference. The final result of the interaction of all markers is a globally 
simpler dynamical system, described by a fewer number of frequencies. It is clear, that based on Inharmonicity Index, gait is naturally described by a harmonic oscillator with high resonance based on the $\mathrm{Q}$ factor. Free limbs are operated at the stride frequency and axial regions at the step frequency. Running is described by a highly harmonic spectrum that has a stronger resonance than walking, therefore the overall coordination is more phase coherent, tightly coupled, efficient, stable, and less complex than walking. The global result of oscillations could provide evidence for central pattern generators based on one oscillator, and that could provide a candidate collective variable for gait.

Future work will examine to what extent the current approach is sensitive to perturbations of the biomechanical and neurological systems, highlighting how redundancy afforded within our motor control can facilitate adaptations. Specific investigations will include the effect of age, injury, and different environmental constraints on gait patterns, in order to increase both theoretical and applied knowledge for human movement science.

\section{References}

Alexander, R. M. N. (2004). Bipedal animals and their differences from humans. Journal of Anatomy, 204, 321-230.

Bernstein, N. A. (1967). The co-ordination and regulation of movements. Oxford: Pergamon Press.

Biewener, A. A., Farley, C. T., Roberts, T.J., \& Temper, M. (2004). Muscle mechanical advantage of human walking and running: implications for energy cost. Journal of Applied Physiology, 97, 2266-2274. 
Blickhan, R. (1989). The spring-mass model for running and hopping. Journal of Biomechanics, 22, 1217-1227.

Bohnsack-McLagan, N. K., Cusumano, J. P., \& Dingwell, J. B. (2016). Adaptability of stride-to-stride control of stepping movements in human walking. Journal of Biomechanics, 49, 229-237.

Cavagna G. A., \& Kaneko, M. (1977). Mechanical work and efficiency in level walking and running. Journal of Physiology. 268,467-481.

Costa, M., Goldberger, A. L., \& Peng. C-K. (2005). Multiscale entropy analysis of biological signals. Physics Reviews E 71, 021906.

Daffertshofer, A., Lamoth, C. J., Meijer, O. G., \& Beek, P. J. (2004). Principal components analysis in studying coordination and variability: a tutorial. Clinical Biomechanics. 19(4), 415-428.

Dingwell, J. B., \& Cusumano, J. P. (2015). Identifying stride-to-stride control strategies in human treadmill walking. PLoS One, 10, e 0124879.

Farley, C. T., \& Ferris, D. P. (1998). Biomechanics of walking and running: centre of mass movements to muscle action. Exercise and Sport Science Reviews, 26, 253-268.

Feynman, R. P., Leighton, R. B., Sands, M. L. (1989). Resonance. In The Feyman Lectures on Physics. Redwood City: Addison-Wesley.

Feynman, R. P., Leighton, R. B., Sands, M. L. (1989a). Harmonics. In The Feyman Lectures on Physics. Redwood City: Addison-Wesley. 
Gage, H. (1964). Accelerographic analysis of human gait. The American Society of Mechanical Engineers. 64, 1-12.

Guertin, P. A. 2012. Central pattern generator for locomotion: Anatomical, physiological, and pathophysiological considerations. Frontiers in Neurology, 3, 183.

Haken, H., Kelso, J. A. S., \& Bunz, H. (1985). A theoretical model of phase transitions in human hand movements. Biological Cybernetics, 51, 347-356.

Kamm, K., Thelen, E., \& Jensen, J. L. (1990). A dynamical systems approach to motor development. Physical Therapy, 70, 763-75.

Kelso, J. A. S. (1995). Dynamic patterns: The self organization of brain and behavior. Cambridge, MA: The MIT Press.

Ko, J. H. \& Newell, K. M. (2015). Organization of postural control patterns as a function of scaling the surface of support dynamics. Journal of Motor Behavior, 47, 415-426.

Kugler, P. N., Kelso, J. A. S., \& Turvey, M. T. (1980). On the concept of coordinative structures as dissipative structures. I. Theoretical lines of 191 convergence. In G. E. Stelmach (Ed.), Tutorials in Motor Behavior. Amsterdam: North-Holland.

Kugler, P. N., \& Turvey, M. T. (1987). Information. natural law. and the self-assembly of rhythmic movement; Theoretical and experimental investigations. Hillsdale. NJ: Erlbaum.

Lamoth, C. J., Daffertshofer, A., Huys, R., \& Beek, P. J. (2009). Steady and transient coordination structures in human walking and running. Human Movement Science, 28, 371-386. 
Lee, C. R., \& Farley, C. T. (1998). Determinants of the center of mass trajectory in human walking and running. The Journal of Experimental Biology, 201, 2935-2944.

Li, L., Haddad, J. M., \& Hamill, J. (2005). Stability and variability may respond differently to changes in walking speed. Human Movement Science, 24, 257-267.

Li, L., van den Bogert, E. C. H., Caldwell, G. E., van Emmerik, R. E. A., \& Hamill, J. (1999). Coordination patterns of walking and running at similar speed and stride frequency. Human Movement Science, 18, 67-85.

Lipsitz, L. A. (2002). Dynamics of stability: the physiologic basis of functional health and frailty. Journal of Gerontology: Biological Science, 57, B115-125.

Lipsitz, L. A., \& Goldberger, A. L. (1992). Loss of 'complexity' and aging: Potential applications of fractals and chaos theory to senescence. Journal of the American Medical Association, 267, 1806-1809.

Newell, K. M. (1985). Coordination, control and skill. In Differing Perspectives in Motor Learning, Memory, and Control, ed. D. Goodman, R. B. Wilberg, I. M. Franks, Amsterdam: Elsevier, 295-317.

Newell, K. M. (1989). On task and theory specificity. Journal of Motor Behavior, 21, 92-96.

Newell, K. M., \& Vaillancourt, D. E. (2001). Dimensional change in motor learning. Human Movement Science, 20(4), 695-715.

Novacheck, T. F. (1998). The biomechanics of running. Gait and Posture 7, 77-95. 
Roche, J.L., Lowry, K.A., Vanswearingen, J.M., Brach, J.S., \& Redfern, M.S. (2013).

Harmonic Ratios: A quantification of step to step symmetry. Journal of Biomechanics, $46,828-831$.

Scholz, J., Kelso, S., \& Schöner, G. (1987). Nonequilibrium phase transitions in coordinated biological motion: critical slowing down and switching time. Physics Letters, 123, 390-394.

Schöner, G., \& Kelso, J. A. S. (1988). Dynamic pattern generation in behavioral and neural systems. Science, 239, 1513-1520.

Schöner, G., Jiang, W. Y., \& Kelso, J. A. S. (1990). A synergetic theory of quadrupedal gaits and gait transitions. Journal of Theoretical Biology, 142, 359-391.

Seay, J. F., Haddad, J. M., van Emmerik, R. E. A., \& Hamill, J. (2006). Coordination variability around the walk to run transition during human locomotion. Motor Control, 10, 178-196.

Segers, V., Aerts, P., Lemoir, M., \& De Clercq, D. (2007a). Dynamics of the body centre of mass during actual acceleration across transition speeds. Journal of Experimental Biology, 210, 578-585.

Segers, V., Aerts, P., Lenoir, M., \& De Clercq, D. (2006). Spatiotemporal characteristics of the walk-to-run and run-to-walk transition when gradually changing speed. Gait and Posture, 24, 247 -254.

Segers, V., Lenoir, M., Aerts, P., \& De Clercq, D. (2007b). Kinematics of the transition between walking and running when gradually changing speed. Gait and Posture, 26, $349-361$. 
Smidt, G.L., Arora, J.S., Johnston, R.C. (1971). Accelerographic analysis of several types of walking. American journal of physical medicine, 50, 285-300.

van Emmerik, R. E. A., Ducharme, S. W., Amanda, A. C., \& Hamill, J. (2016).

Comparing dynamical systems concepts and techniques for biomechanical analysis. Journal of Sport and Health Science, 5, 3-13.

van Emmerik, R. E. A., Miller, R. H., \& Hamill, J. (2014). Dynamical Systems methods for the analysis of movement coordination. In Robertson, G. Et al. (Eds.), Research Methods In Biomechanics ( $2^{\text {nd }}$ edition). Human Kinetics, Champaign, Ill, USA.

Vereijken, B., van Emmerik, R. E. A., Whiting, H. T. A., \& Newell, K. M. (1992). Free(z)ing degrees of freedom in skill acquisition. Journal of Motor Behavior, 24, 133142.

von Holst, E. (1954). Relations between the central nervous system and the peripheral organs. British Journal of Animal Behaviour, 2, 89-94.

Wagenaar, R. C., \& van Emmerik, R. E. (1994). Dynamics of pathological gait. Human Movement Science, 13(3), 441-471.

Wagenaar, R. C., \& Van Emmerik, R. E. A. (2000). Resonant frequencies of arms and legs identify different walking patterns. Journal of Biomechanics, 33(7), 853-861.

Wannier, T., Bastiannse, C., Colombo, G., \& Dietz, V. (2001). Arm to leg coordination during walking, running and creeping activities. Experimental Brain Research, 141, $375-379$. 
Zanone, P. G., \& Kelso, J. A. S. (1997). Coordination dynamics of learning and transfer: Collective and component levels. Journal of Experimental Psychology: Human Perception and Performance, 23, 1454-1480.

\section{Funding}

This research did not receive any specific grant from funding agencies in the public, commercial, or not-for-profit sectors. This research did not receive any specific grant from funding agencies in the public, commercial, or not-for-profit sectors.

\section{Disclosure of Interest}

The authors report no conflict of interest. 
Table 1. Discrete variables quantifying the spectrograms for walking ( $3 \mathrm{~km} / \mathrm{h}$, Figure 2$)$ and running $(15 \mathrm{~km} / \mathrm{h}$, Figure 3$)$. $\mathrm{Q}$ is the resonance quality factor, where a larger number reflects a stronger resonance. Inharmonicity Index is a measurement of deviation of the spectral peaks from a perfectly harmonic spectrum (Equation 1), where a number closer to zero reflect a more harmonic spectrum.

Figure 1. Sonogram of the oscillation of the left (a) and right (b) finger as the velocity of wagging is increased over time ( $\mathrm{x}$ axis). The combination of the left and right fingers is shown in (c). Blue spectral lines represent the highest amplitude oscillation, red regions are related to medium amplitude oscillations and yellow represents the lower energy contribution to the spectrum.

Figure 2. Spectrograms of horizontal components: 2a Top left, left knee marker; $2 b$ top right, left leg (combined left knee, ankle, heel and toe markers); $2 \mathrm{c}$ bottom left, left leg combined with right leg; $2 \mathrm{~d}$ bottom right, whole body (combined left leg, right leg, left arm, right arm, hips, PSIS, shoulder and head markers), when walking at $3 \mathrm{~km} / \mathrm{h}$.

Figure 3. Spectrograms of horizontal components of: 2a Top left, left knee marker; $2 b$ top right, left leg (combined left knee, ankle, heel and toe markers); 2c bottom left, left leg combined with right leg; $2 \mathrm{~d}$ bottom right, whole body (combined left leg, right leg, left arm, right arm, hips, PSIS, shoulder and head markers), when running at $15 \mathrm{~km} / \mathrm{h}$.

Figure 4 . Spectra of the whole body marker combination at $3 \mathrm{~km} / \mathrm{h}$ (grey) and $15 \mathrm{~km} / \mathrm{h}$ (black) having been normalised to step frequency.

Figure 5. Frequency distribution of the spectra of the whole body marker combination at $3 \mathrm{~km} / \mathrm{h}$ (grey) and $15 \mathrm{~km} / \mathrm{h}$ (black) in $2 \mathrm{~Hz}$ bands between $0 \mathrm{~Hz}$ and $12 \mathrm{~Hz}$. 

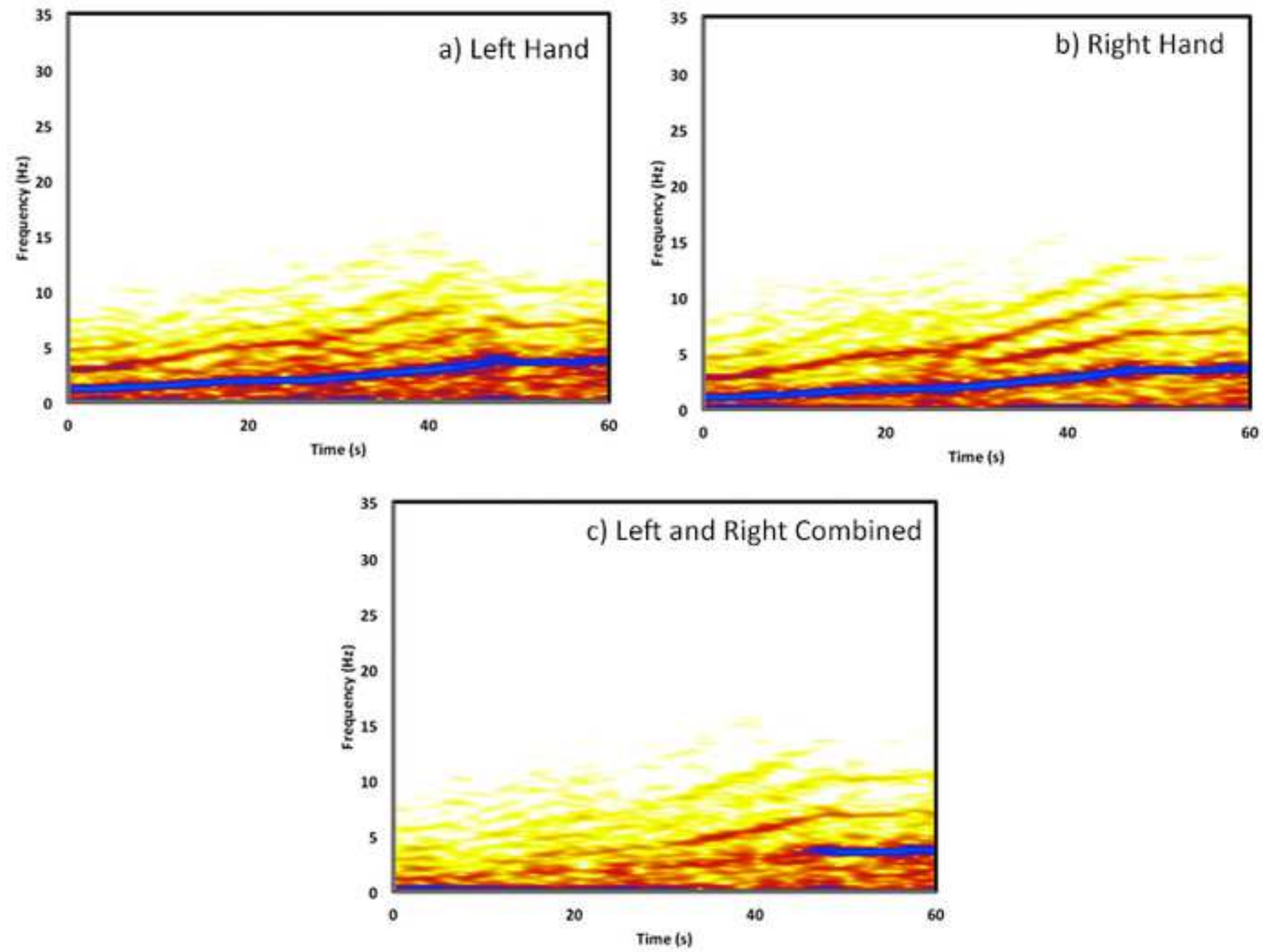

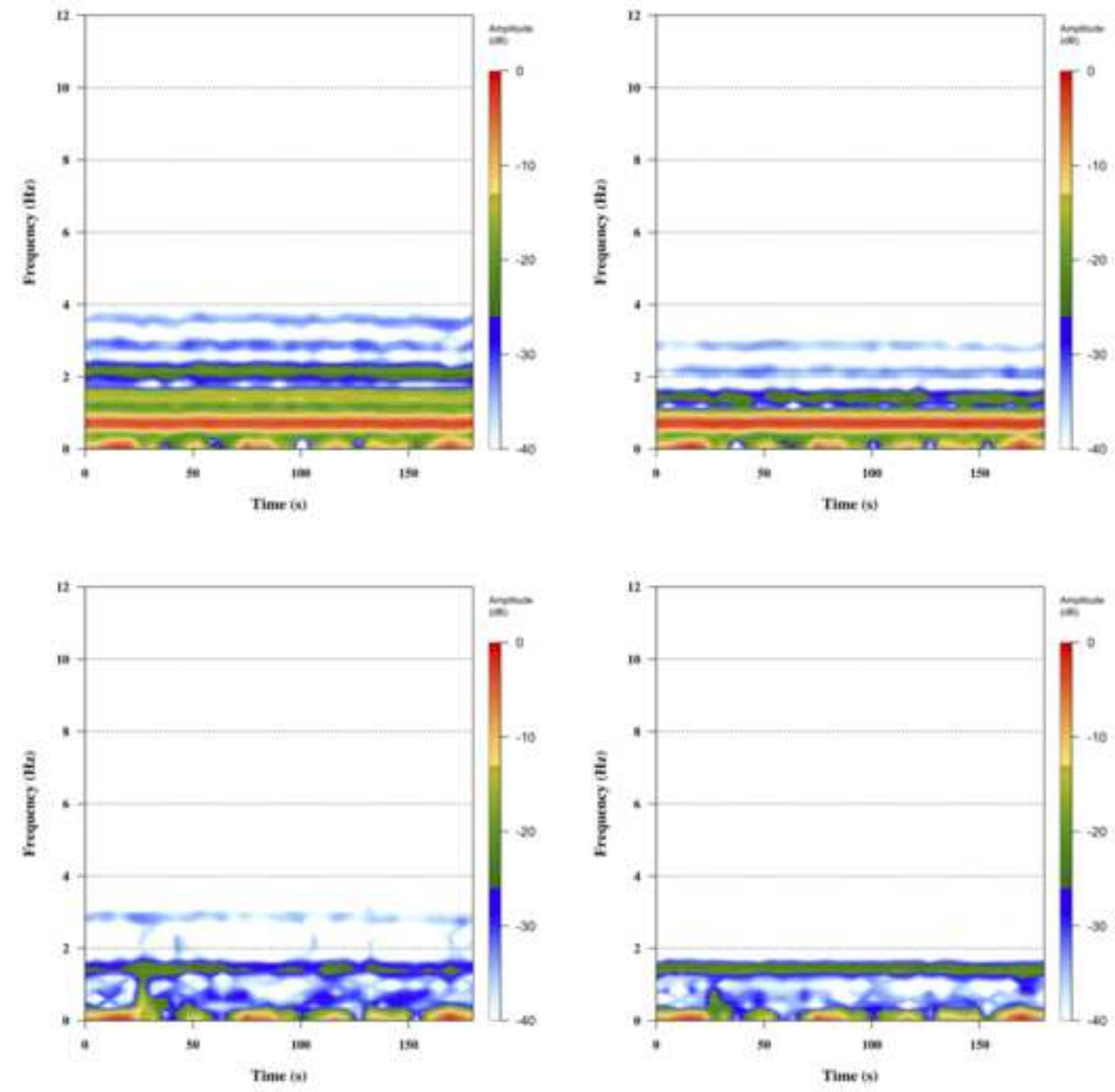

8



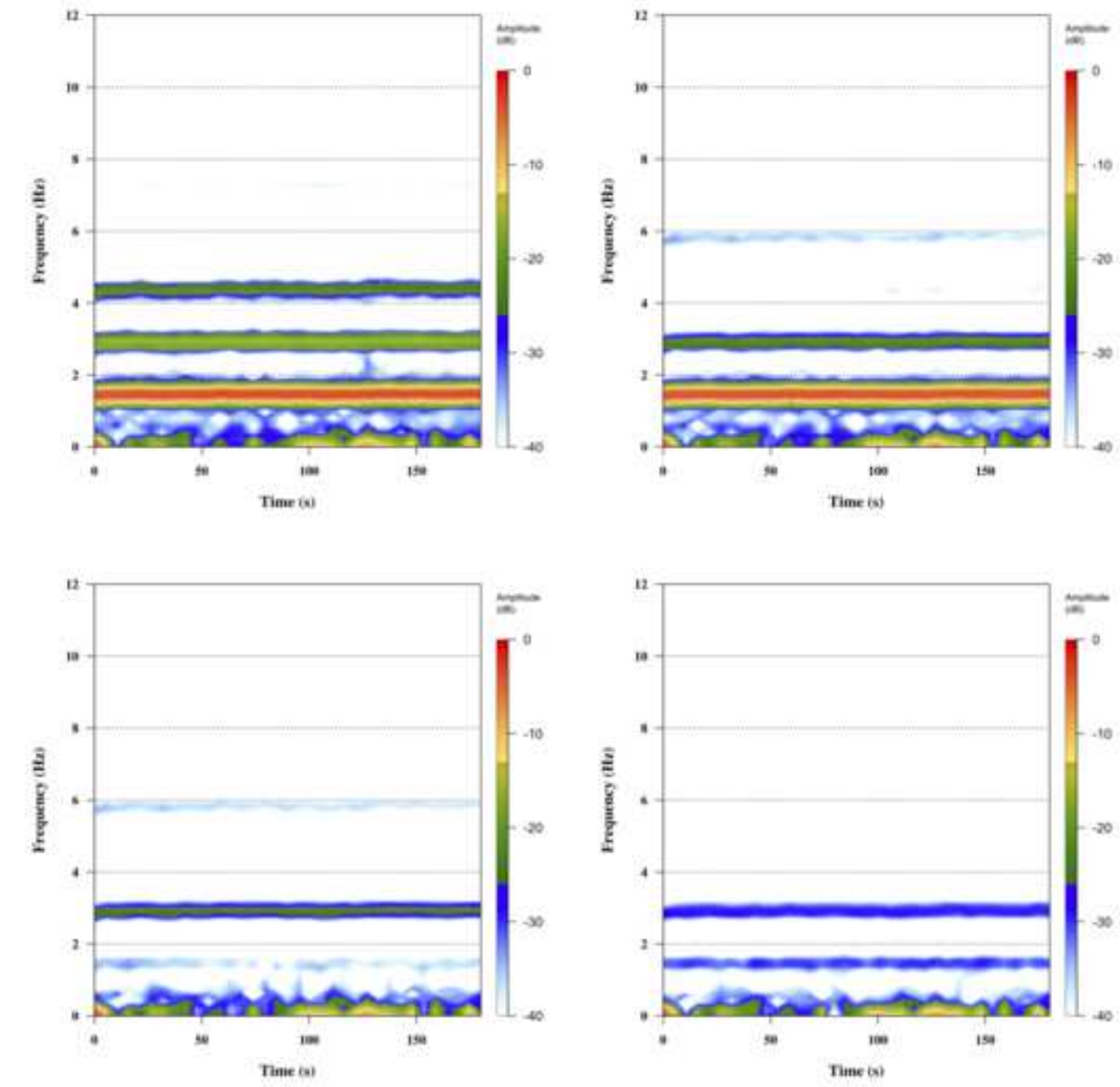

Figure 3

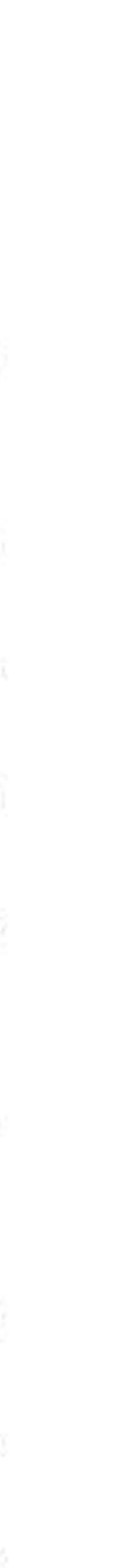

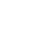
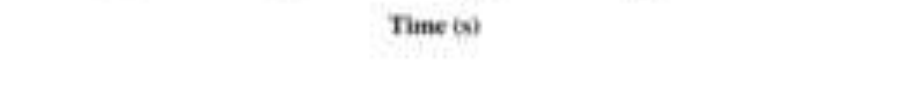


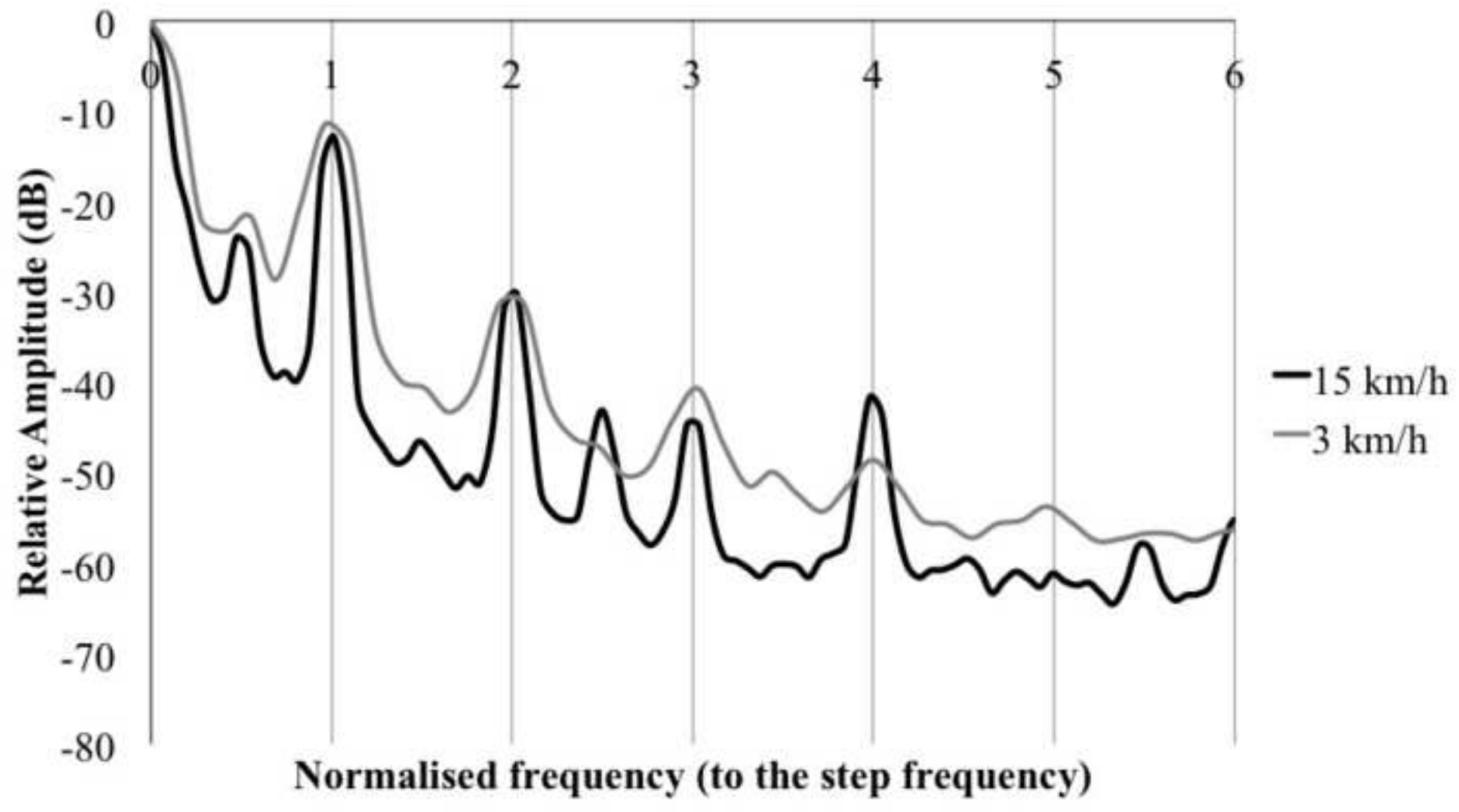




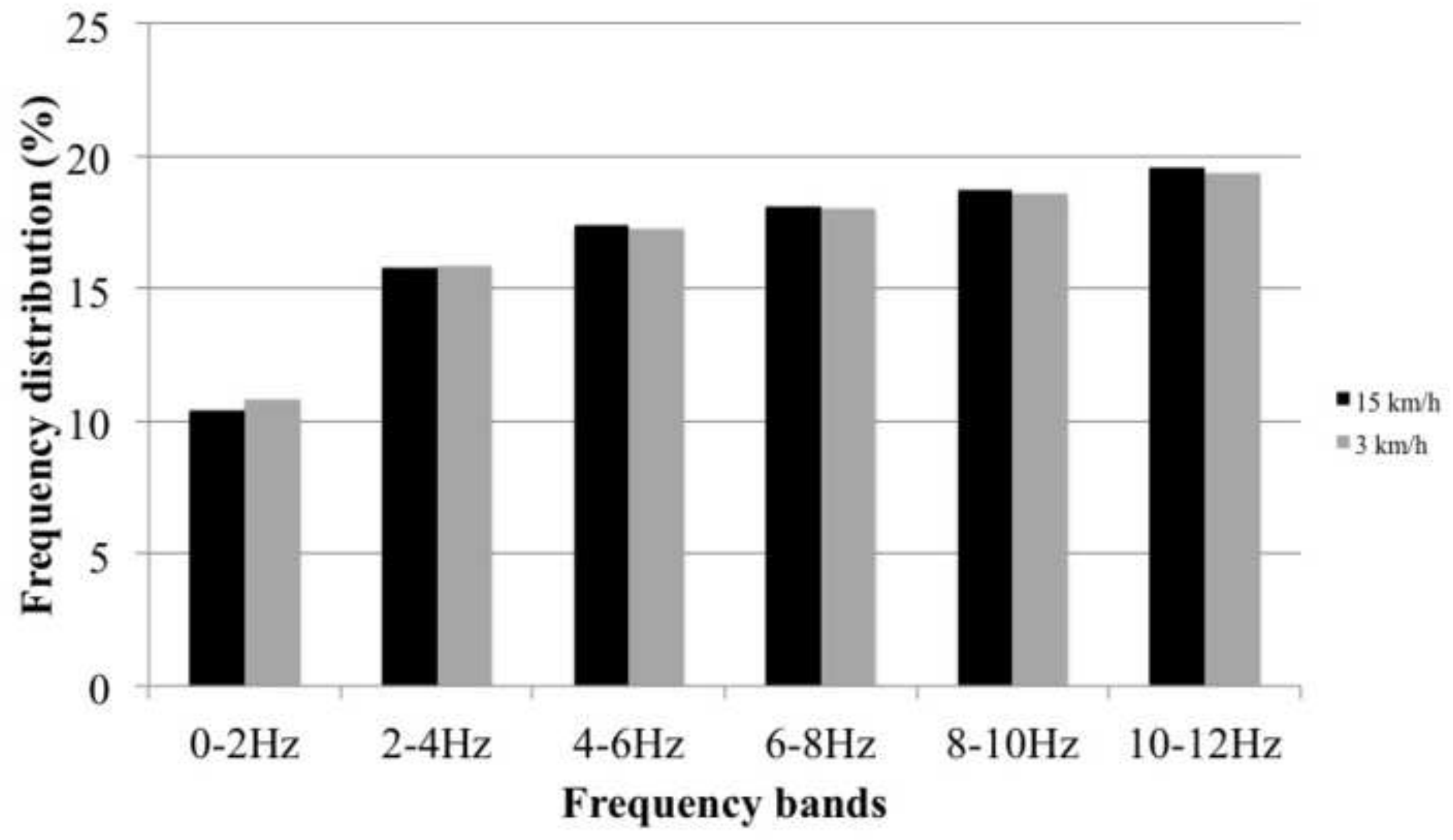




\begin{tabular}{lcccc}
\hline Markers & $\begin{array}{c}\text { Gait velocity } \\
(\mathbf{k m} / \mathbf{h})\end{array}$ & $\begin{array}{c}\text { Base Frequency } \\
\mathbf{( H z )}\end{array}$ & $\mathbf{Q}$ & $\begin{array}{c}\text { Inharmonicity } \\
\text { Index }\end{array}$ \\
\hline Knee & 3 & 0.78 & 2.93 & 0.036 \\
& 15 & 1.37 & 5.11 & 0.007 \\
Leg & 3 & 0.78 & 2.95 & 0.026 \\
& 15 & 1.37 & 5.12 & 0.007 \\
Leg Leg & 3 & 1.37 & 3.04 & 0.102 \\
& 15 & 2.93 & 10.31 & 0.086 \\
Whole & & & & \\
body & 3 & 1.37 & 3.83 & 0.063 \\
& 15 & 2.93 & 11.24 & 0.001 \\
\hline
\end{tabular}

